\title{
On the iterative methods for solving fractional initial value problems: new perspective
}

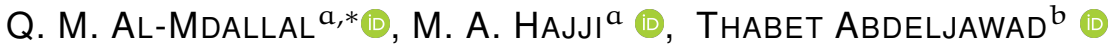 \\ ${ }^{a}$ Department of Mathematical Sciences, United Arab Emirates University, P.O. Box 15551, Al \\ Ain, Abu Dhabi, UAE. \\ ${ }^{b}$ Department of Mathematics and Physical Sciences, Prince Sultan University, P. O. Box \\ 66833, 11586 Riyadh, Saudi Arabia.
}

\author{
- Received: 25.06.2021 • Accepted: 28.06.2021 • Published Online: 29.06.2021
}

\begin{abstract}
In this short communication, we introduce a new perspective for a numerical solution of fractional initial value problems (FIVPs). Basically, we split the considered FIVP into FIVPs on subdomains which can be solved iteratively to obtain the approximate solution for the whole domain.
\end{abstract}

Keywords: Fractional initial value problem, Fractional integral operator, Iterative methods. 2010 MSC: 34A08, 26A33, 65F10.

\section{Introduction}

In recent years, huge interests from scientists in modelling problems in the fields of fluid mechanics, electromagnetic, acoustics, chemistry, biology, physics and material sciences using fractional differential equations (FDEs); see, by way of example not exhaustive enumeration, $[1,2,3,4,5,6,7,8,9]$. However, unlike the integer differential equations (DEs), the determination of the exact solutions of FDEs is so complicated. Therefore, here is a race between researchers to discover accurate, simple and efficient numerical schemes to approximate the exact solutions. For instance, Kazem et al. [10] applied fractional-order Legendre Spectral Galerkin method, Bhrawy and Zaky [11] implemented the shifted fractional-order Jacobi orthogonal functions, and Rehman and Khan [12] implemented the Legendre wavelet method. It should be noted that the fractional-Legendre functions have been implemented in the so-called fractionalLegendre-Galerkin spectral method by few researchers in order to solve several types of fractional ordinary differential equations, see for example Kazem et al. [10], Klimek and Agrawal [13], Bhrawy and Alghamdi [14], Yiming et al. [15], Bhrawy et al. [16], 
Adams-Basforth method [17, 18] and Monotone iterative techniques [25, 24]. It is worth mentioning herein, that all the above numerical schemes are implemented on the whole domain which creates accumulative error that may significantly affects the accuracy and simplicity of numerical schemes. Consequently, our target is to handle this problem by proposing a new iterative technique that allow us to solve the FIDEs on subdomains. The explanation of this method will applied on the following fractional initial value problems of the form:

$$
D_{a}^{\alpha} y(t)=f(t, y(t)) \quad t \in(a, T], 0<\alpha \leqslant 1,
$$

subject to

$$
y(a)=h_{a},
$$

where $h_{a} \in \mathbb{R}$ and $y \in L_{1}(a, T)$. The notation $D_{a}^{\alpha}$ denotes the fractional derivative. Without loss of generality, we assume $\mathrm{D}_{\mathrm{a}}^{\alpha}$ be the left-sided fractional derivative of Caputo sense.

\section{Preliminary Results}

In this section, we present some basic definitions and properties of fractional calculus theory.

Definition 2.1. The left-sided Riemann-Liouville fractional integral operator of order $\alpha$ is defined by

$$
J_{a}^{\alpha} y(t)=\frac{1}{\Gamma(\alpha)} \int_{a}^{t}(t-\tau)^{\alpha-1} y(\tau) d \tau, \quad \alpha \in \mathbb{R},
$$

where, $t \in[a, T], y$ belongs to the Lebesgue space $L_{1}[a, T]$ and $\Gamma$ is the Euler gamma function defined by

$$
\Gamma(\alpha)=\int_{0}^{\infty} s^{\alpha-1} e^{-s} \mathrm{~d} s .
$$

Lemma 2.2. ([19, 20, 21]) Let $\alpha, \beta>0, t \in[a, T], \gamma>-1$ and $y \in L_{1}[a, T]$. Then

(i) $J_{a}^{0} y(t)=y(t)$,

(ii) $J_{a}^{\alpha} J_{a}^{\beta} y(t)=J_{a}^{\alpha+\beta} y(t)=J_{a}^{\beta} J_{a}^{\alpha} y(t)$.

Definition 2.3. For $\alpha \in \mathbb{R}, \mathrm{m}=\lceil\alpha\rceil$ and $\mathrm{t} \in[\mathrm{a}, \mathrm{T}]$, the left-sided Caputo fractional derivatives operator is defined as:

$$
D_{a}^{\alpha} y(t)=J_{a}^{m-\alpha} y^{(m)}(t)=\frac{1}{\Gamma(m-\alpha)} \int_{a}^{t}(t-\tau)^{m-\alpha-1} y^{(m)}(\tau) d \tau,
$$

provided the integral exists. This operator was introduced by the Italian mathematician Caputo in 1967, see [22].

Lemma 2.4. For $\alpha \in \mathbb{R}, \mathrm{m}=\lceil\alpha\rceil, \mathrm{t} \in[\mathrm{a}, \mathrm{T}]$ and $\mathrm{y} \in \mathrm{L}_{1}[\mathrm{a}, \mathrm{T}]$, we have

1. $D_{a}^{\alpha} J_{a}^{\alpha} y(t)=y(t)$.

2. $J_{a}^{\alpha} D_{a}^{\alpha} y(t)=y(t)-\sum_{k=0}^{m-1} y^{(k)}(a) \frac{t^{k}}{k !}$. 


\section{Main results}

In this section, for the sake of showing the motivation of our method, we solve the fractional differential equation .

$$
D_{a}^{\alpha} y(t)=f(t, y(t)), y(a)=h_{a}, t \in I:=[a, T], \alpha \in(0,1)
$$

The domain $[a, T]$ is divided into $N$-subintervals with the grid points $t_{n}=a+n h, n=$ $0, \cdots, N$. Here $h$ represents a uniform step size; $h=(T-a) / N$. Hence, the domain can be written as $I=\bigcup_{n=1}^{N} I_{n}$ where $I_{n}=\left[t_{n-1}, t_{n}\right]$. Write the solution in the domain decomposition form $y(t)=\sum_{n=1}^{N} y_{n}(t)$, where

$$
y_{n}(t)= \begin{cases}y(t), & t \in I_{n} \\ 0, & \text { o.w. }\end{cases}
$$

It should be noted herein that since $\alpha \in(0,1]$, we assume the continuity of the solution at the nodes $t_{n-1}$, i.e., $y_{n}\left(t_{n-1}\right)=y_{n-1}\left(t_{n-1}\right)$ for all $n \geqslant 2$. The following Lemma is the heart of the motivation in this work.

Lemma 3.1. For $\mathrm{t} \in \mathrm{I}_{\mathrm{n}}$, equation (3.1) reduces to

$$
D_{t_{n-1}}^{\alpha} y_{n}(t)=f\left(t, y_{n}(t)\right), y_{n}\left(t_{n-1}\right)= \begin{cases}y_{n-1}\left(t_{n-1}\right), & n \geqslant 2 \\ y_{0}, & n=1\end{cases}
$$

Proof. Since $t \in I_{n}$, then based on the restrictions on $y(t)(3.2), y(t)=y_{n}(t)$. In addition, applying the continuity condition at $t=t_{n-1}$, the results is obtained.

As a result of Lemma 1, we may easily convert (3.3) to integral equation as shown in the next lemma.

Lemma 3.2. For $\mathrm{t} \in \mathrm{I}_{\mathrm{n}}$, the FIVP (3.3) on sub-domain $\mathrm{I}_{\mathrm{n}}$ is equivalent to the following integral equation:

$$
y_{n}(t)=y_{n-1}\left(t_{n-1}\right)+\frac{1}{\Gamma(\alpha)} \int_{t_{n-1}}^{t}(t-\tau)^{\alpha-1} f\left(\tau, y_{n}(\tau)\right) d \tau
$$

Proof. Applying the integral operator $J_{\mathfrak{t}_{n-1}}^{\alpha}$ to both sides of equation (3.3), one obtains

$$
\begin{aligned}
J_{t_{n-1}}^{\alpha} D_{t_{n-1}}^{\alpha} y_{n}(t) & =J_{t_{n-1}}^{\alpha} f\left(t, y_{n}(t)\right) \\
y_{n}(t)-y_{n}\left(t_{n-1}\right) & =\frac{1}{\Gamma(\alpha)} \int_{t_{n-1}}^{t}(t-\tau)^{\alpha-1} f\left(\tau, y_{n}(\tau)\right) d \tau .
\end{aligned}
$$

Using the continuity condition at the node $t_{n-1}$, i.e., $y_{n}\left(t_{n-1}\right)=y_{n-1}\left(t_{n-1}\right)$, the result is obtained. 
The benefits of the present algorithm can be easily observed especially in the large domains. Additionally, it can be applied for any numerical technique. In the next section we will present one example to show the efficiency of the present work.

A simple algorithm can be derived from the integral equation (3.4) is by approximating the function $f\left(\tau, y_{n}(\tau)\right)$ at the point $t=t_{n-1}$; i.e., $f\left(\tau, y_{n}(\tau)\right) \approx f\left(t_{n-1}, y_{n-1}\left(t_{n-1}\right)\right)$. Consequently, the integral equation (3.4) will be given by

$$
\begin{aligned}
y_{n}(t) & =y_{n-1}\left(t_{n-1}\right)+\frac{f\left(t_{n-1}, y_{n-1}\left(t_{n-1}\right)\right)}{\Gamma(\alpha)} \int_{t_{n-1}}^{t}(t-\tau)^{\alpha-1} d \tau \\
& =y_{n-1}\left(t_{n-1}\right)+\frac{f\left(t_{n-1}, y_{n-1}\left(t_{n-1}\right)\right)}{\alpha \Gamma(\alpha)}\left(t-t_{n-1}\right)^{\alpha} \\
& =y_{n-1}\left(t_{n-1}\right)+\frac{f\left(t_{n-1}, y_{n-1}\left(t_{n-1}\right)\right)}{\Gamma(\alpha+1)}\left(t-t_{n-1}\right)^{\alpha} .
\end{aligned}
$$

Notice that replacing $t$ by $t_{n}$ will lead to the well-known algorithm, fractional explicit Euler method [23]. On the other hand, we can easily obtain a fractional implicit method at $t=t_{n}$ by approximating $f(\tau, y(\tau))$ in the integral equation (3.4) using the nodes $t_{n-1}$ and $t_{n}$ :

$$
f\left(\tau, y_{n}(\tau)\right) \approx \frac{1}{h}\left[\left(\tau-t_{n-1}\right) f\left(t_{n}, y_{n}\left(t_{n}\right)\right)-\left(\tau-t_{n}\right) f\left(t_{n-1}, y_{n}\left(t_{n-1}\right)\right)\right]
$$

Therefore, (3.4) will be converted to

$$
y_{n}(t)=y_{n-1}\left(t_{n-1}\right)+\frac{h^{\alpha}}{\Gamma(\alpha+2)}\left(\alpha f\left(t_{n-1}, y_{n-1}\left(t_{n-1}\right)\right)+f\left(t_{n}, y_{n}\left(t_{n}\right)\right)\right)
$$

\section{Concluding Remarks and Future work}

The present work deals with numerical treatment of fractional initial value problems with large domains. we showed that this problem can be solved iteratively in selected sub-domains. Derivation of explicit and implicit fractional methods is illustrated.

\section{Acknowledgment}

The authors would like to express their appreciation for the valuable comments of the reviewers. The authors also would like to express their sincere appreciation to the United Arab Emirates University, Al Ain, UAE for providing the financial support with the grant 31S363-UPAR (4) 2018.

\section{References}

[1] Dehghan M, Hamedi EA and Khosravian-Arab H (2016). A numerical scheme for the solution of a class of fractional variational and optimal control problems using the modified Jacobi polynomials. Journal of Vibration and Control, 22(6): 1547-1559.

[2] Ortigueira MD, Ionescu CM, Machado JT and Trujillo JJ (2015). Fractional signal processing and applications. Signal Processing, 107(C): 197. https://doi.org/10.1016/j.sigpro.2014.10.002 
[3] Kulish VV and Lage JL (2002). Application of fractional calculus to fluid mechanics. J. Fluids Eng., 124(3): 803-806. https:/ / doi.org/10.1115/1.1478062

[4] Verotta D (2010). Fractional dynamics pharmacokinetics-pharmacodynamic models. Journal of pharmacokinetics and pharmacodynamics, 37(3): 257-276.

[5] Gafiychuk V, Datsko B and Meleshko V (2008). Mathematical modeling of time fractional reaction-diffusion systems. Journal of Computational and Applied Mathematics, 220(1-2): 215-225. https:/ / doi.org/10.1016/j.cam.2007.08.011

[6] Al-Mdallal QM and Hajji MA (2015). A convergent algorithm for solving higher-order nonlinear fractional boundary value problems. Fractional Calculus and Applied Analysis, 18(6): 1423-1440. https:/ / doi.org/10.1515/fca-2015-0082

[7] Al-Mdallal QM, Yusuf $\mathrm{H}$ and Ali A (2020). A novel algorithm for time-fractional foam drainage equation. Alexandria Engineering Journal, 59(3): 1607-1612. https://doi.org/10.1016/j.aej.2020.04.007

[8] Ahmad S, Ullah A, Al-Mdallal QM, Khan H, Shah K and Khan A (2020). Fractional order mathematical modeling of COVID-19 transmission. Chaos, Solitons \& Fractals, 139: 110256. https:/ / doi.org/10.1016/j.chaos.2020.110256

[9] Aman S, Abdeljawad T and Al-Mdallal QM (2020). Natural convection flow of a fluid using Atangana and Baleanu fractional model. Advances in Difference Equations, 2020(1): 1-15. https://doi.org/10.1186/s13662-020-02768-w

[10] Kazem S, Abbasbandy S and Kumar S (2013). Fractional-order Legendre functions for solving fractional-order differential equations. Applied Mathematical Modelling, 37(7): 5498-5510. https://doi.org/10.1016/j.apm.2012.10.026

[11] Bhrawy AH and Zaky MA (2016). Shifted fractional-order Jacobi orthogonal functions: application to a system of fractional differential equations. Applied Mathematical Modelling, 40(2): 832-845. https:/ / doi.org/10.1016/j.apm.2015.06.012

[12] ur Rehman M and Khan RA (2011). The Legendre wavelet method for solving fractional differential equations. Communications in Nonlinear Science and Numerical Simulation, 16(11): 4163-4173. https:/ / doi.org/10.1016/j.cnsns.2011.01.014

[13] Klimek M and Agrawal OP (2013). Fractional Sturm-Liouville problem. Computers \& Mathematics with Applications, 66(5): 795-812.

[14] Bhrawy AH and Alghamdi MA (2013 January). A new legendre spectral galerkin and pseudo-spectral approximations for fractional initial value problems. In Abstract and Applied Analysis, 2013: Article ID 306746. https: / / doi.org/10.1155/2013/306746

[15] Chen Y, Sun Y and Liu L (2014). Numerical solution of fractional partial differential equations with variable coefficients using generalized fractional-order Legendre functions. Applied Mathematics and Computation, 244: 847-858. https://doi.org/10.1016/j.amc.2014.07.050

[16] Bhrawy AH, Taha TM and Machado JAT (2015). A review of operational matrices and spectral techniques for fractional calculus. Nonlinear Dynamics, 81(3): 1023-1052.

[17] Toufik M and Atangana A (2017). New numerical approximation of fractional derivative with non-local and non-singular kernel: application to chaotic models. The European Physical Journal Plus, 132(10): 1-16. https:/ / doi.org/10.1140/epjp/i2017-11717-0

[18] Rihan FA, Al-Mdallal QM, AlSakaji HJ and Hashish A (2019). A fractional-order epidemic model with time-delay and nonlinear incidence rate. Chaos, Solitons \& Fractals, 126: 97-105. https:/ / doi.org/10.1016/j.chaos.2019.05.039

[19] Oldham K and Spanier J (1974). The fractional calculus theory and applications of differentiation and integration to arbitrary order. Elsevier.

[20] Miller KS and Ross B (1993). An introduction to the fractional calculus and fractional differential equations. Wiley.

[21] Podlubny I (1998). Fractional differential equations: an introduction to fractional derivatives, fractional differential equations, to methods of their solution and some of their applications. Elsevier.

[22] Caputo M (1967). Linear models of dissipation whose $Q$ is almost frequency independent-II. Geophysical Journal International, 13(5):529-539.

[23] Odibat ZM and Momani S (2008). An algorithm for the numerical solution of differential equations of fractional order. Journal of Applied Mathematics \& Informatics, 26: 15-27.

[24] Shah K, Shah L, Ahmad S, Rassias JM and Li Y (2019). Monotone iterative techniques together with Hyers-Rassias stability. Mathematical Methods in the Applied Sciences, 44(10). https:/ / doi.org/10.1002/mma.5825 
[25] Al-Refai M and Hajji MA (2011). Monotone iterative sequences for nonlinear boundary value problems of fractional order. Nonlinear Analysis: Theory, Methods \& Applications, 74(11): 3531-3539. https:/ / doi.org/10.1016/j.na.2011.03.006 